\title{
Review Article \\ Effects of Exercise-Induced ROS on the Pathophysiological Functions of Skeletal Muscle
}

\author{
Fan Wang $\mathbb{B}$, Xin Wang $\mathbb{D}$, Yiping Liu $\mathbb{D}$, and Zhenghong Zhang $\mathbb{D}$ \\ Provincial University Key Laboratory of Sport and Health Science, Provincial Key Laboratory for Developmental Biology \\ and Neurosciences, Key Laboratory of Optoelectronic Science and Technology for Medicine of Ministry of Education, School of \\ Physical Education and Sport Sciences, Fujian Normal University, Fuzhou 350007, China
}

Correspondence should be addressed to Yiping Liu; ypliu1966@fjnu.edu.cn and Zhenghong Zhang; zhangzh@fjnu.edu.cn

Received 16 August 2021; Accepted 18 September 2021; Published 1 October 2021

Academic Editor: Yanqing Wu

Copyright () 2021 Fan Wang et al. This is an open access article distributed under the Creative Commons Attribution License, which permits unrestricted use, distribution, and reproduction in any medium, provided the original work is properly cited.

Oxidative stress is the imbalance of the redox system in the body, which produces excessive reactive oxygen species, leads to multiple cellular damages, and closely relates to some pathological conditions, such as insulin resistance and inflammation. Meanwhile, exercise as an external stimulus of oxidative stress causes the changes of pathophysiological functions in the tissues and organs, including skeletal muscle. Exercise-induced oxidative stress is considered to have different effects on the structure and function of skeletal muscle. Long-term regular or moderate exercise-induced oxidative stress is closely related to the formation of muscle adaptation, while excessive free radicals produced by strenuous or acute exercise can cause muscle oxidative stress fatigue and damage, which impacts exercise capacity and damages the body's health. The present review systematically summarizes the relationship between exercise-induced oxidative stress and the adaptions, damage, and fatigue in skeletal muscle, in order to clarify the effects of exercise-induced oxidative stress on the pathophysiological functions of skeletal muscle.

\section{Introduction}

Exercise as a common stressor leads to oxygen supply that cannot meet a rapid increase in oxygen demand of the body; then, many tissues and organs produce some highly active molecules, such as reactive nitrogen species (RNS) and reactive oxygen species (ROS). ROS plays a pivotal role in the stress process, including superoxide anion $\left(\mathrm{O}_{2}{ }^{--}\right)$, hydroxyl radical $\left(\mathrm{OH}^{--}\right)$, and hydrogen peroxide $\left(\mathrm{H}_{2} \mathrm{O}_{2}\right)$ (Figure 1) [1]. The production of ROS exceeds its scavenging capacity, which is the reaction of oxidative stress. Under normal physiological conditions, ROS participates in a number of cell activities, including cell energy metabolism, signal transduction, and gene expression regulation, but high levels of ROS can also damage biomacromolecules in cells, such as lipids, proteins, and nucleic acids, leading to cell senescence even death [2]. A large number of studies have shown that regular or suitable exercise produces low-level ROS, while excessive production of endogenous free radicals during exercise can damage the physiological functions of the entire tissue [1-3], such as skeletal muscle [3]. Skeletal muscle is the dynamic part of the exercise system, and the physiological level ROS is an essential substance for maintaining its function, which is involved in the production of muscle force, the maintenance of muscle content, intracellular signal transduction, gene expression, and other related activities, while excessive ROS causes the dysfunction of contraction and muscle weakness [4]. As is well known, ROS induced by skeletal muscle contraction during exercise can increase the level of oxidative stress and enhance the antioxidant defense system. Although exercise-induced reactive oxygen species are required for normal force production in skeletal muscle, the high levels of ROS can contribute to contractile dysfunction [5]. The sarcoplasmic reticulum $\mathrm{Ca}^{2+}$ release channel is highly sensitive to ROS, which will reduce the sensitivity of myofibrils to $\mathrm{Ca}^{2+}$ and then affect muscle contraction [5, 6]. In addition, the accumulation of ROS in the body depends on exercise mode, exercise intensity, and duration. 


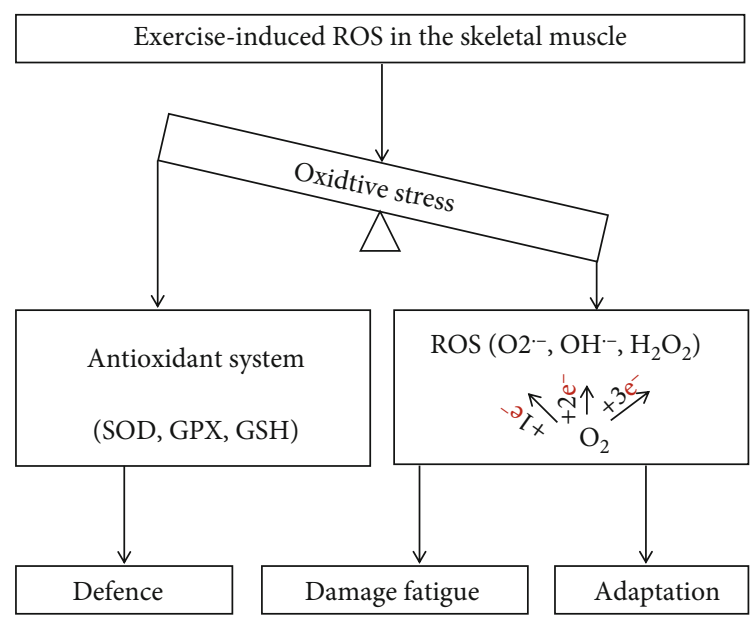

Figure 1: Effects of exercise-induced ROS on the balance of oxidative stress in the skeletal muscle. Exercise-induced oxidative stress occurs as the production of ROS generated in the body, which is excessive to the defense ability of antioxidant system during physical exercise. ROS includes $\mathrm{O}_{2}{ }^{-}, \mathrm{OH}^{-}$, and $\mathrm{H}_{2} \mathrm{O}_{2}$, which affect the adaption, damage, and fatigue in the skeletal muscle.

Therefore, exercise-induced oxidative stress may play an important role in the pathophysiological functions of skeletal muscle.

\section{Exercise-Induced Oxidative Stress}

In 1978, exercise-induced oxidative stress was firstly proposed, which refers to oxidative stress caused by exercise; that is, the rate of free radicals generated in the body is much greater than its scavenging rate during physical exercise, which will damage tissue, resulting in the decline of the body's working ability [7]. The mitochondrial respiratory chain is the main source of endogenous ROS during exercise, and its excessive production can cause oxidative damage to lipids, proteins, nucleic acids, and other substances [8]. The accumulation of ROS in the body depends on the exercise mode, intensity, and duration. Studies have indicated that a small amount of ROS produced by moderateintensity exercise can act as a second messenger in cells to mediate growth factor signal transmission [9-11]. Acute exercise can promote the excessive production of ROS, which causes an imbalance in the oxidation-antioxidant homeostasis in cells, because rapidly increasing oxygen consumption inevitably produces more free radicals during strenuous exercise [12]. A large amount of oxygen is consumed to produce a large amount of singlet oxygen $\left(\mathrm{O}_{2}{ }^{--}\right)$, which stimulates a series of radical chain reactions [13]. On the other hand, the hypoxia of local tissue and the accumulation of metabolites affect the mitochondrial energy supply. Meanwhile, high-intensity exhaustive exercise can cause a significant increase in the content of malondialdehyde (MDA) and further increase with the extension exercise [14]. MDA can objectively reflect the level of free radicals in the body.

\section{ROS in Skeletal Muscle}

Skeletal muscle is an important exercise organ in the body; its normal physiological function is the basic prerequisite and important guarantee for health.

3.1. ROS in Different Skeletal Muscle Fibers. Skeletal muscle is composed of three types of muscle fibers: I, IIa, and IIb. The abilities of these different types of muscle fibers to generate ROS and resist oxidative stress are also different [15]. The leakage level of mitochondrial ROS in type IIb muscle fiber is 2-3 times that of type IIa muscle fiber, due to mitochondria in type IIb muscle fiber playing an important role in the production and release of superoxide [4]. High-intensity exercise leads to the excessive production of ROS, which can cause changes in the normal physiological environment of skeletal muscle fibers and vascular endothelial dysfunction. The levels of antioxidant enzymes of type I and IIa muscle fibers enhance the scavenging ability of free radicals and promote the recovery of skeletal muscle motor functions, while there is no significant change in type IIb muscle fibers. In addition, type I muscle fibers are rich in myoglobin with the strongest abilities of aerobic metabolism and antifatigue [16]. These findings indicate that different types of muscle fibers have different effects on antifatigue and ROS production, but they are all related to their antioxidant capacity.

3.2. Sites for ROS in Skeletal Muscle Cells. In the past, mitochondria are the main site of intracellular ROS in contracting skeletal muscle [14]. In mitochondria, $2-5 \%$ of the total oxygen consumed may undergo one electron reduction with the generation of superoxide [17]. Mitochondrial complex I in the electron transport chain releases superoxide to the mitochondrial matrix, while complex III to both sides of the inner membrane [18]. Recently, some scholars have discovered that the main source of ROS may not be limited to mitochondria during exercise [14]. The upper limit of the total utilization of oxygen consumed by mitochondria in different tissues to produce ROS is about $0.15 \%$ [19]. Simultaneously, a large number of studies have shown that many intracellular enzymes are involved in the production of ROS, such as NADPH oxidases (NOXs), xanthine oxidase (XO), and phospholipase A2 (PLA2) [18]. Regardless of whether muscle is in a resting or contracting state, NOX family proteins can produce more superoxide anions than that of mitochondria in single muscle fibers [20]. Moreover, the NOX2 loss-offunction model indicated that NOX2 may be the main source of cytosolic ROS in skeletal muscle during moderate-intensity exercise [21]. Another study found that there was a correlation between XO content and lactic acid level during anaerobic exercise, producing a large amount of ROS, thereby aggravating skeletal muscle oxidative damage [22]. In addition, muscle damage caused by exercise can also stimulate various cytokines to activate macrophages and neutrophils, leading to the overproduction of ROS [23].

\section{Skeletal Muscle Adaptation}

Skeletal muscle is a highly plastic tissue. Moderate or regular exercise training can regulate the signaling pathways by 


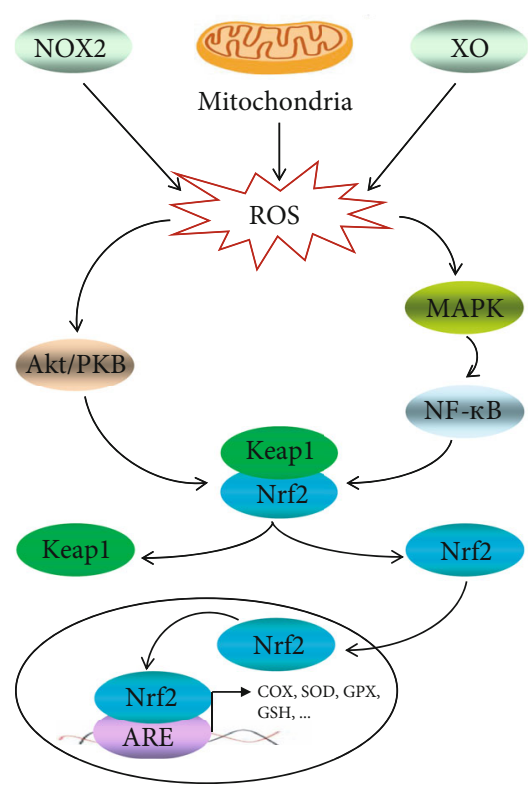

FIGURE 2: Effects of exercise-induced ROS on the signaling pathway in the skeletal muscle. Exercise-induced ROS can activate MAPK and Akt signaling pathways. Nrf2 dissociates from its cytoplasmic inhibitor Keap1 then translocates to the nucleus and combines with ARE to contribute to some downstream antioxidant gene transactivation, such as the oxidases, COX, SOD, GPX, and GSH.

oxidative stress and ROS to resist oxidative damage and further come true structure-function adaptations in skeletal muscle $[19,24,25]$. ROS can not only activate NF- $\kappa$ B by activating mitogen-activated protein kinase (MAPK) but also stimulate Akt phosphorylation, thereby activating their common downstream molecule nuclear factor erythroidderived 2-related factor 2 (Nrf2) [25]. Nrf2 as a redoxsensing transcription factor dissociates from its cytoplasmic inhibitor Keap1, then translocates to the nucleus and combines with antioxidant response element (ARE) to contribute to transactivation of some downstream antioxidant genes, especially defense-related enzymes and exerciseadapting-related enzymes, such as oxidase cytochrome oxidase (COX), superoxide dismutase (SOD), glutathione peroxidase (GPX) activities, and glutathione (GSH), thereby upregulating their expressions and activities, alleviating the oxidative damage, and promoting exercise-induced adaptations (Figure 2) [19, 24, 25]. Acute exercise or long-term exercise training may increase the expression of Nrf2 and/or Nrf2-ARE binding activity. After 6 weeks of treadmill training, the transcription activity of Nrf2 in skeletal muscle is increased, and the activity of COX is also increased by $20 \%$ [26].

Moreover, exercise-activated Nrf2 may regulate skeletal muscle mitochondria by promoting mitochondrial biogenesis, improving mitochondrial respiratory function, and regulating mitochondrial autophagy. In addition, peroxisome proliferator-activated receptor- $\gamma$ coactivator- $1 \alpha$ (PGC- $1 \alpha$ ) plays an important role in glucose uptake, mitochondriogenesis, and hypertrophy in skeletal muscle responses to physical exercise [24]. Exercise-induced ROS can couple with lactate metabolism to stimulate PGC- $1 \alpha$ production, espe- cially endurance exercise [24]. PGC- $1 \alpha$ coactivates nuclear respiratory factor- (NRF-) 1 and NRF-2 to increase their DNA binding in skeletal muscle after acute exercise [24], then activates the genes encoding COX and mitochondrial transcription factor A (TFAM), which activate mitochondrial DNA transcription, thereby increasing mitochondrial synthesis [24]. In addition, exercise-induced ROS are able to stimulate the skeletal muscle to secrete myokines, which also play important roles in the regulation of cell signaling and muscle metabolic adaptation [27, 28], such as interleukin-15 (IL-15). IL-15 is a regulator to control intracellular ROS production and attenuate oxidative stress in skeletal muscle cells [29].

\section{Skeletal Muscle Cell Damage}

Exhaustive exercise can increase the peroxidation and weaken the antioxidant capacity in skeletal muscles, which can cause the damage of skeletal muscle cells through modification of lipid, protein, and DNA.

Firstly, for lipid damage, biomembrane is the main component of cells. Lipid peroxidation caused by excessive endogenous ROS during exercise can change the liquidity, fluidity, and permeability of the biomembrane, which in turn leads to membrane dysfunction [30]. The lipid bilayer contains a large amount of polyunsaturated fatty acid (PUFA). ROS can deprive the hydrogen at the diallyl position in PUFA to form unsaturated fatty acid free radicals, then combine with oxygen molecules to generate lipid peroxyl radicals $\mathrm{LOO}^{-}$[31]. LOO- can deprive the allyl groups of unsaturated fatty acid hydrogen which forms a new unsaturated fatty acid free radical and lipid peroxide (LOOH), causing oxidative damage to the biomembranes of skeletal muscle cells [31].

Secondly, for protein damage, ROS plays a very important role in the process of protein metabolism, which can promote protein turnover and renewal under physiological conditions. But excessive ROS induced by exercise will cause damage to proteins and induce the inactivation of enzymes, the loss of receptors, and the decline of immune function [31]. The damage of proteins by ROS is mainly to change their activities. $\mathrm{OH}^{-}$can change the primary structure of the protein and contribute to the secondary and tertiary structure modification, so that the polypeptide chain unfolds to form a random structure [32]. These modifications expose the originally shielded peptide bonds to proteolytic enzymes. In addition, a protein is combined with $\mathrm{OH}^{-}$which will increase the production of double tyrosine, further induce the breakage, which is manifested by the effect of extracting hydrogen on the a-carbon atom of the amino acid, and then react with $\mathrm{O}_{2}{ }^{--}$to generate $\mathrm{LOO}^{-}$, ultimately forming peroxides [32].

The last is DNA damage. 8-Hydroxydeoxyguanosine (8OHdG) is an important marker for the detection of oxidative DNA damage. The C-8 position of the base guanine in the DNA chain is susceptible to the attack of $\mathrm{OH}^{-}$and $\mathrm{O}_{2}{ }^{-}$ and undergoes hydroxylation to form the adduct 8-OHdG [33]. $\mathrm{OH}^{-}$combines with the deoxyguanine nucleotide residues at the C-4, C-5, or C-8 positions of DNA bases to form 
8-hydroxy-7,8-dihydroxyguanine nucleotides, which in turn further oxidizes to generate 8-hydroxydeoxyguanine nucleotides. Simultaneously, during the replication process, 8OHdG on the DNA chain can pair with other bases than $\mathrm{C}$ to form point mutations, such as $\mathrm{GC} \rightarrow \mathrm{TA}$ [32].

\section{Skeletal Muscle Fatigue}

The process of sports fatigue is a complex physiological phenomenon; it actually occurs with the consumption of physical energy or the accumulation of metabolites, including skeletal muscle fatigue, visceral fatigue, and nerve fatigue. Skeletal muscle fatigue is the main peripheral manifestation of sports fatigue. The production of skeletal muscle strength depends on its contraction mechanisms, while any disorder of the nerves, ions, blood vessels, and energy systems upstream of the cross bridge will lead to their loss and cause muscle fatigue, especially the energy metabolism factors during the process of muscle contraction, such as $\mathrm{H}^{+}$, lactic acid, $\mathrm{Pi}$, reactive ROS, heat shock protein (HSP), $\alpha$-acid glycoprotein (ORM) which also affect muscle fatigue.

Excessive ROS produced by strenuous exercise may cause exercise fatigue by reducing the release of skeletal muscle sarcoplasmic reticulum $\mathrm{Ca}^{2+}$ and/or the sensitivity of myofibrils $\mathrm{Ca}^{2+}$ [34]. However, under mild oxidative stress induced by physical exercise, the S-glutathionization of troponin can improve contractile apparatus $\mathrm{Ca}^{2+}$ sensitivity in fast-twitch fibers, thereby delaying the occurrence of fatigue [35]. However, antioxidants restored $\mathrm{Ca}^{2+}$ to release in the sarcoplasmic reticulum, but the decrease in muscle strength during fatigue cannot be eliminated [36], indicating that oxidative stress caused by exercise is closely related to muscle fatigue. Endogenous and exogenous ROS not only may hinder muscle fiber contraction by affecting the release and uptake of $\mathrm{Ca}^{2+}$ in the sarcoplasmic reticulum and reducing the activity of troponin but also can promote the occurrence of exercise fatigue by destroying mitochondrial functions and inhibiting aerobic metabolism [6]. In addition, during exercise, the increased energy metabolism rate leads to excessive mitochondrial ROS production in fatigued skeletal muscle cells, which further leads to the occurrence of the oxidative modification of lipids, proteins, and DNA.

\section{Conclusion}

Together, exercise-induced oxidative stress has dual effects on skeletal muscle tissue. Proper exercise can promote generation of the physiological levels of ROS, maintain the normal skeletal muscle function, and facilitate exercise adaptation, while exhaustive exercise can cause the body to produce too much ROS, which causes excessive oxidative stress, leading to fatigue and cell damage of skeletal muscle. Therefore, further understanding the effect of exerciseinduced oxidative stress on the pathophysiological functions of skeletal muscle is very important for guiding exercise to promote health and provides a direction to explore the molecular mechanism of exercise to improve the development of pathophysiological processes in oxidative stressrelated diseases.

\section{Data Availability}

The original contributions presented in the study are included in the article. Further inquiries can be directed to the corresponding authors.

\section{Conflicts of Interest}

The authors declare that they have no competing interests.

\section{Authors' Contributions}

The work was conceived by FW, XW, YL, and ZZ; draft writing was performed by FW and XW; and the manuscript was revised by YL and ZZ. All authors reviewed and approved the final version of the manuscript for publication.

\section{Acknowledgments}

This work was supported by the Key Projects of Scientific and Technological Innovation in Fujian Province (2021G02021 and 2021G02023), Special Funds of the Central Government Guilding Local Science and Technology Development (2020L3008), Fujian Provincial Natural Science Foundation (2019R1011-3 and 2020J01176), and Scientific Research Talent Project of Fujian Provincial Health Commission (2018-ZQN-20).

\section{References}

[1] S. K. Powers, R. Deminice, M. Ozdemir, T. Yoshihara, M. P. Bomkamp, and H. Hyatt, "Exercise-induced oxidative stress: friend or foe?," Journal of Sport and Health Science, vol. 9, no. 5, pp. 415-425, 2020.

[2] Z. He, Q. Xu, B. Newland et al., "Reactive oxygen species (ROS): utilizing injectable antioxidative hydrogels and ROSproducing therapies to manage the double-edged sword," Journal of Materials Chemistry B, vol. 9, no. 32, pp. 6326-6346, 2021.

[3] S. K. Powers, W. B. Nelson, and M. B. Hudson, "Exerciseinduced oxidative stress in humans: cause and consequences," Free Radical Biology \& Medicine, vol. 51, no. 5, pp. 942-950, 2011.

[4] A. Thirupathi, R. A. Pinho, and Y.-Z. Chang, "Physical exercise: an inducer of positive oxidative stress in skeletal muscle aging," Life Sciences, vol. 252, 2020.

[5] F. Magherini, T. Fiaschi, R. Marzocchini et al., "Oxidative stress in exercise training: the involvement of inflammation and peripheral signals," Free Radical Research, vol. 53, no. 11-12, pp. 1155-1165, 2019.

[6] A. Cheng, T. Yamada, D. E. Rassier, D. C. Andersson, H. Westerblad, and J. T. Lanner, "Reactive oxygen/nitrogen species and contractile function in skeletal muscle during fatigue and recovery," The Journal of Physiology, vol. 594, no. 18, pp. 5149-5160, 2016.

[7] M. C. Fogarty, C. M. Hughes, G. Burke et al., "Exerciseinduced lipid peroxidation: implications for deoxyribonucleic acid damage and systemic free radical generation," Environmental and Molecular Mutagenesis, vol. 52, no. 1, pp. 35-42, 2011. 
[8] A. Sarniak, J. Lipińska, K. Tytman, and S. Lipińska, "Endogenous mechanisms of reactive oxygen species (ROS) generation," Postępy Higieny I Medycyny Doświadczalnej, vol. 70, pp. 1150-1165, 2016.

[9] C. R. Reczek and N. S. Chandel, "ROS-dependent signal transduction," Current Opinion in Cell Biology, vol. 33, pp. 8-13, 2015.

[10] M. C. Gomez-Cabrera, J. Viña, and L. L. Ji, "Role of redox signaling and inflammation in skeletal muscle adaptations to training," Antioxidants, vol. 5, no. 4, p. 48, 2016.

[11] M.-C. Gomez-Cabrera, C. Borrás, F. V. Pallardó, J. Sastre, L. L. Ji, and J. Viña, "Decreasing xanthine oxidase-mediated oxidative stress prevents useful cellular adaptations to exercise in rats," The Journal of Physiology, vol. 567, no. 1, pp. 113-120, 2005.

[12] G. Schippinger, W. Wonisch, P. M. Abuja, F. Fankhauser, B. Winklhofer-Roob, and G. Halwachs, "Lipid peroxidation and antioxidant status in professional American football players during competition," European Journal of Clinical Investigation, vol. 32, no. 9, pp. 686-692, 2002.

[13] S. G. Sokolovski, E. U. Rafailov, A. Y. Abramov, and P. R. Angelova, "Singlet oxygen stimulates mitochondrial bioenergetics in brain cells," Free Radical Biology and Medicine, vol. 163, pp. 306-313, 2021.

[14] S. Joanisse, J. McKendry, C. Lim et al., "Understanding the effects of nutrition and post-exercise nutrition on skeletal muscle protein turnover: insights from stable isotope studies," Clinical Nutrition Open Science, vol. 36, pp. 56-77, 2021.

[15] S. K. Powers, Z. Radak, and L. L. Ji, "Exercise-induced oxidative stress: past, present and future," Journal of Physiology, vol. 594, no. 18, pp. 5081-5092, 2016.

[16] L. Zhang, Y. Zhou, W. Wu et al., "Skeletal muscle-specific overexpression of PGC- $1 \alpha$ induces fiber-type conversion through enhanced mitochondrial respiration and fatty acid oxidation in mice and pigs," International Journal of Biological Sciences, vol. 13, no. 9, pp. 1152-1162, 2017.

[17] S. K. Powers and M. J. Jackson, "Exercise-induced oxidative stress: cellular mechanisms and impact on muscle force production," Physiological Reviews, vol. 88, no. 4, pp. 1243-1276, 2008.

[18] E. L. Robb, A. R. Hall, T. A. Prime et al., "Complex I reverse electron transport," Journal of Biological Chemistry, vol. 293, no. 25, pp. 9869-9879, 2018.

[19] P. Steinbacher and P. Eckl, "Impact of oxidative stress on exercising skeletal muscle," Biomolecules, vol. 5, no. 2, pp. 356-377, 2015.

[20] G. K. Sakellariou, A. Vasilaki, J. Palomero et al., "Studies of mitochondrial and nonmitochondrial sources implicate nicotinamide adenine dinucleotide phosphate oxidase(s) in the increased skeletal muscle superoxide generation that occurs during contractile activity," Antioxidants \& Redox Signaling, vol. 18, no. 6, pp. 603-621, 2013.

[21] C. Henríquez-Olguin, J. R. Knudsen, S. H. Raun et al., "Cytosolic ROS production by NADPH oxidase 2 regulates muscle glucose uptake during exercise," Nature Communications, vol. 10, no. 1, p. 4623, 2019.

[22] M.-C. Gomez-Cabrera, E. Domenech, and J. Viña, "Moderate exercise is an antioxidant: upregulation of antioxidant genes by training," Free Radical Biology \& Medicine, vol. 44, no. 2, pp. 126-131, 2008.
[23] P. Baumert, E. C. Hall, and R. M. Erskine, "The genetic association with exercise-induced muscle damage and muscle injury risk," in Sports, Exercise, and Nutritional Genomics, pp. 375407, Elsevier, 2019.

[24] J. Bouviere, R. S. Fortunato, C. Dupuy, J. P. Werneck-de-Castro, D. P. Carvalho, and R. A. Louzada, "Exercise-stimulated ROS sensitive signaling pathways in skeletal muscle," Antioxidants, vol. 10, no. 4, p. 537, 2021.

[25] P. M. Abruzzo, F. Esposito, C. Marchionni et al., "Moderate exercise training induces ROS-related adaptations to skeletal muscles," International Journal of Sports Medicine, vol. 34, no. 8, pp. 676-687, 2013.

[26] M. J. Crilly, L. D. Tryon, A. T. Erlich, and D. A. Hood, “The role of Nrf2 in skeletal muscle contractile and mitochondrial function," Journal of Applied Physiology, vol. 121, no. 3, pp. 730-740, 2016.

[27] C. Scheele, S. Nielsen, and B. K. Pedersen, "ROS and myokines promote muscle adaptation to exercise," Trends in Endocrinology and Metabolism, vol. 20, no. 3, pp. 95-99, 2009.

[28] J. Starnes, T. Parry, S. O’Neal et al., "Exercise-induced alterations in skeletal muscle, heart, liver, and serum metabolome identified by non-targeted metabolomics analysis," Metabolites, vol. 7, no. 3, pp. 40-54, 2017.

[29] F. Li, Y. Li, Y. Tang et al., "Protective effect of myokine IL-15 against $\mathrm{H}_{2} \mathrm{O}_{2}$-mediated oxidative stress in skeletal muscle cells," Molecular Biology Reports, vol. 41, no. 11, pp. 7715$7722,2014$.

[30] F. Ito, Y. Sono, and T. Ito, "Measurement and clinical significance of lipid peroxidation as a biomarker of oxidative stress: oxidative stress in diabetes, atherosclerosis, and chronic inflammation," Antioxidants, vol. 8, no. 3, pp. 72-100, 2019.

[31] U. N. Das, "Exercise is beneficial: but how and why?," Circulation Journal, vol. 75, no. 4, pp. 1010-1011, 2011.

[32] A. Vasilaki, A. Richardson, H. Van Remmen et al., "Role of nerve-muscle interactions and reactive oxygen species in regulation of muscle proteostasis with ageing," The Journal of Physiology, vol. 595, no. 20, pp. 6409-6415, 2017.

[33] H. Kasai, N. Iwamoto-Tanaka, T. Miyamoto et al., "Life style and urinary 8-hydroxydeoxyguanosine, a marker of oxidative DNA damage: effects of exercise, working conditions, meat intake, body mass index, and smoking," Japanese Journal of Cancer Research, vol. 92, no. 1, pp. 9-15, 2001.

[34] J. Ying, X. Cen, and Y. Peimin, "Effects of eccentric exercise on skeletal muscle injury: from an ultrastructure aspect: a review," Physical Activity and Health, vol. 5, no. 1, pp. 15-20, 2021.

[35] J. Osório Alves, L. Matta Pereira, I. Cabral Coutinho do Rêgo Monteiro et al., "Strenuous acute exercise induces slow and fast twitch-dependent NADPH oxidase expression in rat skeletal muscle," Antioxidants, vol. 9, no. 1, p. 57, 2020.

[36] L. Delgado-Roche, N. Lagumersindez, and G. Martínez-Sánchez, "Biochemical changes in mitochondria and its role in cell death during myocardial ischemia-reperfusion injury," Pharmacologyonline, vol. 2, 2009. 